\title{
LA PRODUZIONE LETTERARIA DI MARISA MADIERI
}

\section{Corinna Gerbaz GiUliano}

Riassunto: Marisa Madieri inaugura con Verde acqua il filone della letteratura dell'esodo al femminile. La memoria costituisce il perno della narrazione e il tema dell'esilio rappresenta una costante nella scrittura madieriana. Il linguaggio, caratterizzato da un registro espressivo intenso e puro, e dall'essenzialità dell'espressione, solo all'apparenza si presenta semplice e accessibile, ma scava in profondità, in una dialettica in cui passato e presente si rifrangono e risolvono in una pacata, luminosa conciliazione.

Limpidezza e semplicità nell'espressione artistica contraddistinguono la voce discreta e raffinata di Marisa Madieri (Fiume 1938 - Trieste 1996), approdata al mondo letterario nella seconda metà degli anni ottanta. Dopo l'esodo dalla città natia nel 1949, Madieri si è trasferita a Trieste dove si è costruita una vita. In seguito a momenti difficili che hanno segnato la sua esistenza da esule, si è inserita nel tessuto sociale triestino: conseguita la laurea, ha lavorato dapprima alle Assicurazioni Generali e poi quale insegnante d'inglese in vari licei triestini. Un aspetto fondamentale della vita di Madieri è costituito dall'opera di volontariato che ha svolto con passione per diversi anni. Trieste è stata inoltre sinonimo d'amore e famiglia: dal matrimonio con Claudio Magris, affermato germanista e scrittore, sono nati due figli, Paolo e Francesco. Cristina Benussi fa notare come al di là dei temi trattati in Verde acqua (1987) — l'infanzia, la famiglia, l'esodo, l'esperienza del campo profughi a Trieste, la conquista di una nuova dimensione individuale e famigliare - sia assolutamente doveroso rimarcare lo spirito di gratitudine che emerge dalla scrittura della Madieri nei confronti di Trieste, la città che l'ha accolta ${ }^{1}$.

L'aspetto decisivo del suo percorso di vita è racchiuso nell'esperienza drammatica dell'esodo che non è stata relegata in un canto dell'animo; l'autrice ha vissuto il suo dramma e l'ha elaborato allontanandovi ogni tipo di rancore. Nelle pagine di Marisa Madieri la storia viene rivisitata, ma rimane però ai margini della narrazione; prevale l'elemento intimo, quello del microcosmo familiare da cui traspare l'aspetto plurimo dell'identità di

1 Per ulteriori delucidazioni si rimanda al saggio di Cristina Benussi "Storie e memorie letterarie", 31-65. 
frontiera, un'identità vissuta nella piena consapevolezza di appartenere a una società multietnica. Il bisogno di sentirsi ancora legata alla terra d'origine è quindi una necessità reale da cui la scrittrice non fugge: lo testimoniano le ripetute visite della famiglia Madieri/Magris alla bellissima costa istriana, ai boschi della Slovenia, alle isole del Quarnero, tra cui spiccano Cherso e Lussino e l'amata Miholaščica, nonché l'esigenza di imparare nuovamente la lingua croata.

La sua attività letteraria, "originale, straordinariamente ricca di rimandi, di sguardi, di voci, di storie plurali, che intersecano le inquietudini della cultura contemporanea, e della quale a Marisa Madieri va riconosciuta senz'altro la primogenitura" (Benussi, 176), inizia con il romanzo Verde acqua, uscito nel 1987. Seguono la raccolta di racconti La radura $(1987)^{2} \mathrm{e}$ le pubblicazioni postume La conchiglia e altri racconti (1998) e Maria (2007). Pubblica inoltre vari racconti e testi d'impegno sociale tra cui Aprile (1990), Il bambino con le ali (1992), Notte d'estate (1994), I barattoli (1995), Riccardo e la sirena (1996), Acqua è poesia - Water is poetry (1989), "Due voci sulla liberazione della donna. Aborto sì, aborto no. Discussione con Franca Ongaro Basaglia" (1982), "Emarginazione a Trieste: famiglia" (1989), "Introduzione a 'Ambito A — Gruppo di studio 4 - Accoglienza della vita nascente e iniziative per una efficace cultura della vita: associazionismo, volontariato e chiesa locale"' (1990).

Ermanno Paccagnini sostiene che la narrativa di Marisa Madieri può essere definita

come singolare esperienza letteraria, anomala, fuori dagli schemi consolidati $[. .$.$] fuori da mode e correnti. Quindici anni di creatività narrati-$ va che producono un totale di 250 pagine circa, tra racconti ed interventi di vario tipo e i due esili volumi Verde acqua e La radura, donati a distanza di anni l'uno dall'altro, e di scrittura, forma e architettura assai diverse (d'un altro romanzo, Maria, restano invece abbozzi, appunti e alcuni capitoli). (Paccagnini, V)

Nel suo saggio Paccagnini riporta le significative parole di Vittore Branca, che definisce i libri della Madieri all'"acqua e sapone", un appellativo questo che rispecchia di fatto la sua scrittura, ma "d'un 'sapone' che rivendica un'insigne marca stilistica: e d'un'acqua' di sorgiva trasparenza” (Paccagnini, V). L'acqua è un elemento costante della scrittura madieriana, che significa limpidezza dell'animo, trasparenza che lascia intravvedere pure il lato oscuro della vita, ma anche mare cristallino, quel mare bellissi-

2 Vincitore del Premio Costantino Pavan nel 1993. 
mo che non divide, ma unisce e avvicina popoli e culture in un disegno universale, dove il passato non si cancella, non si rimuove, bensì viene amalgamato dentro a un concetto di cittadinanza in cui il rapporto tra identità e diversità non viene vissuto con senso di esclusione e dove la diversità non diventa pregiudizio.

La bibliografia critica sull'opera di Marisa Madieri è molto corposa: la compongono, infatti, circa trecento tra saggi e articoli. Tra gli strumenti critici più rilevanti vanno ricordate le attente e dettagliate letture di Graziano Benelli, Graziano Bianchi, Ermanno Paccagnini, Maria Carminati, Ernestina Pellegrini.

Nel 1987, quando esordisce con il libro Verde acqua nella collana Einaudi - Nuovi Coralli, inaugurando di fatto il filone femminile della narrativa sull'esodo, riscontra un immediato successo. Secondo Maria Carminati, Marisa Madieri può essere considerata "capostipite di questo filone che racconta l'esilio degli istriani e dalmati con emozione priva di rancore" (Carminati, "Marisa Madieri”, 177). Apprezzato dalla critica, il romanzo viene tradotto in varie lingue: croato, francese, spagnolo, tedesco, inglese, polacco, sloveno (altre traduzioni sono in corso).

In Verde acqua, romanzo in forma di diario, racchiuso in un arco temporale che va dal 24 novembre 1981 al 27 novembre 1984, la scrittrice narra la drammatica vicenda dell'esodo, la sua odissea, che è al tempo stesso l'odissea dei profughi che hanno abbandonato la loro terra d'origine. Nel romanzo però, passato e presente si alternano, si fondono. Non c'è successione lineare nel tempo della narrazione. Lautrice ripercorre il periodo infantile fiumano per poi tuffarsi nel presente triestino della maturità, rivelando al lettore le sue riflessioni più intime, le sue inquietudini, le sue paure, come quella che si riferisce alla scoperta della malattia che la strapperà prematuramente all'affetto dei suoi cari. In questo continuo scambio di sequenze temporali si evince la necessità di narrare una parte di storia poco conosciuta, ma lo scopo non è quello di farsi portavoce di tutti gli esiliati, bensì di narrare la propria storia attraverso la memoria.

L'esodo degli italiani dell'Istria e di Fiume fu un fenomeno di lunga durata che si protrasse dal 1943 fino alla fine degli anni cinquanta, e le conseguenze che produsse furono drammatiche. Nel maggio del 1945, a conclusione del secondo conflitto mondiale, i territori di Fiume, dell'Istria e della Dalmazia entrarono a far parte della Repubblica Socialista Federativa di Jugoslavia, ma problemi e tensioni relativi ai nuovi confini rimasero irrisolti fino al 1947, quando venne firmato il Trattato di pace con l'Italia. La tensione con l'Italia si aggravò ulteriormente in seguito alla crisi apertasi nel 1953 per la questione di Trieste, risolta l'anno seguente con il 
Memorandum di Londra. La popolazione italiana che era rimasta nei territori passati alla Jugoslavia visse momenti di terribile incertezza e intanto si diffondeva l'eco della fuga. Trauma, desolazione, smarrimento, irrigidimento, sradicamento sono solamente alcuni dei tasselli di questo drammatico mosaico, le cui difficoltà maggiori furono vissute sul piano dell'identità e del tortuoso cammino per la sua riconquista individuale e collettiva.

Sul versante letterario questo trauma ebbe vaste ripercussioni: da una parte gli autori esuli, in un primo momento, si concentrarono sullo sradicamento dalle proprie origini usando toni nostalgici, comprensibili e legittimi, in cui l'idea di terra natia veniva rievocata come una sorta di Eden perduto (e va osservato sin d'ora che questa considerazione non è valida nel caso specifico di Marisa Madieri). D'altra parte i rimasti conducevano le loro battaglie per il mantenimento della propria identità etnica e culturale in un clima politico, ideologico e culturale in cui la componente italiana veniva etichettata come non grata. C'era poi una terza 'fazione' di cui facevano parte coloro che si assoggettarono al regime. Ciò che comunque accomunava gli italiani rimasti in Jugoslavia e gli esuli erano la lingua, la cultura e l'identità collettiva attraverso la memoria.

Il concetto di letteratura dell'esodo viene spesso inserito nel contesto della letteratura di confine, termine che oggi sembra cozzare con il linguaggio dell'Europa unita. È un dato di fatto che la letteratura fiumana, istriana, giuliana e dalmata del Novecento sia stata trascurata dal pubblico e dalla critica proprio perché considerata "di confine", nonostante i nomi illustri che la rappresentano, tra cui: Slataper, Stuparich, Quarantotti Gambini, Tomizza, Madieri, Vegliani, Morovich, Santarcangeli, Ramous, Scotti, Schiavato, Mori, Milani e altri.

La letteratura italiana dell'area istro-quarnerina può essere distinta in due periodi che seguono rispettivamente il primo e il secondo conflitto mondiale. Il secondo periodo si colloca storicamente dopo la firma del Trattato di pace del 1947 e il Memorandum d'intesa di Londra del 1954 tra l'Italia e l'allora Federazione jugoslava, ratificato nel 1975 dal Trattato di Osimo. In questo periodo si sviluppano due tipi di produzione letteraria: la prima è quella che si può definire la "letteratura dell'esodo", nata lungo il confine orientale dell'Italia, rappresentata da autori che hanno vissuto in Istria e a Fiume fino al 1945 e che hanno lasciato la loro terra d'origine stabilendosi in Italia e in altri paesi, europei e non. Un esempio illustre di questa letteratura è Fulvio Tomizza, che pur avendo optato appena nel 1955 per l'Italia e avendo scelto Trieste come sua dimora, ha però continuato a recarsi nella natia Materada, quasi per sottolineare questa sua innata convivenza con tre culture e tradizioni diverse: italiana, croata e slovena. 
Contemporaneamente si è venuta a creare l'altra produzione letteraria, al di là del confine orientale, precisamente nel territorio istro-quarnerino, la cosiddetta "letteratura dei rimasti". Per gli autori di confine, sia esuli che rimasti, si è trattato di un impegno sofferto, di una vera e propria scelta di identità, condizionata sì dalla storia, ma intrisa di quella necessità sempre presente di tramandare ai posteri la propria lingua, le proprie tradizioni e la propria cultura, fornendo una valida risorsa all'intera società plurietnica. In questo senso il discorso letterario si è inserito nel gioco delle relazioni interculturali, assumendo un ruolo decisivo all'interno dello scambio dialettico fra le etnie, e in questo modo ha sollecitato quella curiosità verso l'altro che è il naturale presupposto di ogni intesa.

Bruno Maier sostiene che

la letteratura istriana del dopoguerra non può essere interamente compresa sotto l'etichetta riduttiva "letteratura dell'esodo", né è in alcun modo identificabile con essa: al contrario la "letteratura dell'esodo" è una sua parte e componente, e sia pure quella più cospicua e rilevante. Meglio allora parlare, in sede storica e critica, di una letteratura e cultura istriana al di qua del confine con la Jugoslavia (ora con la ex Jugoslavia, ossia con la Slovenia); e di una letteratura e cultura italiana al di là del medesimo confine o, se si preferisce, "istro-quarnerina", "istro-fiumana". (Maier, 81)

$\mathrm{Al}$ di là del diverso destino che vede la medesima popolazione divisa da un confine, si assiste comunque a una comunione di motivi legati alla memoria e al ricordo. Non si riscontrano, a nostro parere, delle fondamentali diversità all'interno della memoria collettiva della popolazione in questione, in quanto la tematica dell'esodo viene vissuta in maniera simile da Fulvio Tomizza, come da Osvaldo Ramous, personalità di spicco della minoranza italiana, rimasto a vivere in Jugoslavia, oppure da Anna Maria Mori e Marisa Madieri — che hanno lasciato la loro terra d'origine - o Nelida Milani, che come Ramous non ha mai abbandonato la sua terra natale. Il dramma dello sradicamento dalle proprie origini ha infatti la stessa intensità a prescindere dalla realtà territoriale in cui viene espresso. Oltre allo spostamento materiale nello spazio e nel tempo, il percorso tematico dell'esilio diviene ricerca e trasformazione interiore. La ricerca è indirizzata alla riscoperta di quei codici culturali d'identità che lo sradicamento ha messo a repentaglio, ma il percorso dell'esilio si attua anche in coloro che non si spostano dalla loro terra d'origine, per cui si verifica - per usare le parole della Milani — "l'esilio interiore". La nuova realtà statuale, venutasi a creare con la nascita della Jugoslavia, produce smarrimento, sradicamento anche tra coloro che scelgono di restare nelle loro città natali e la 
ricerca di sé, che si manifesta nel recupero delle proprie tradizioni, diviene allora l'unico mezzo per combattere l'oblio, diventa lo strumento indispensabile per re-impossessarsi dei parametri identitari.

Il recupero avviene in termini simili anche per chi se n'è andato, per chi è stato costretto ad abbandonare la propria casa e i propri affetti, trovandosi in breve tempo privato della propria identità e dignità umana, catapultato dentro a un'altra identità, quella del profugo. Dopo un iniziale smarrimento, lingua, cultura, espressione dialettale, usi e costumi ridiventano elementi determinanti della costruzione (o ri-costruzione) della propria identità attraverso la memoria individuale e collettiva.

Nel racconto-diario di Marisa Madieri la memoria costituisce il perno della narrazione e rappresenta il "trampolino di lancio [...] che attraverso atti, fatti, sentimenti ed emozioni della quotidianità conduce per mano Marisa Madieri nel turbinio degli anni trascorsi, alla ricerca delle proprie origini (che scopre essere multiple: croate, slovene, ungheresi, italiane)" (Mitrović, 194). Il tema dell'esodo in Marisa Madieri "scioglie i nodi e esplora sentieri percorsi dissodando l'argilla delle radici in riflessi, immagini, simboli di una realtà crudele, poi stemperata in una serenità placante" (Bianchi, 21).

Le considerazioni di Maria Carminati gettano ulteriore luce sul modo di raccontare l'esodo di Marisa Madieri: "l'esilio viene raccontato attraverso un nuovo sguardo, quello dell'alterità, di un soggetto femminile che si trasforma nel corso della narrazione, e che all'interno dell'alterità del soggetto narrante femminile sviluppa una ulteriore differenza, quella del soggetto dell'infanzia" (Carminati, "Marisa Madieri", 181). Proprio in base a queste riflessioni possiamo considerare la scrittura di Verde acqua una scrittura di tipo filosofico, che mira a scrutare il mondo e la vita da un'angolatura inaspettata. Secondo il filosofo francese Roland Barthes non vi è altro luogo d'appartenenza se non quello dell'infanzia. L'idea di Barthes è rintracciabile nella maggior parte degli scritti sull'esodo, in cui questo luogo d'infanzia assume immediatamente una caratteristica peculiare: da una parte è il luogo delle proprie origini salde e ancorate, è l'angolo degli affetti, ma allo stesso tempo è il luogo dello sradicamento, della separazione, è l'effetto del trauma dell'esodo. La tesi di Barthes trova conferma nelle pagine della Madieri, in cui l'infanzia a Fiume costituisce un punto fermo nella scrittura di Verde acqua. Scorrono nella memoria dell'autrice i ricordi della casa della nonna paterna: "Papà e mamma, per motivi economici, erano andati ad abitare a casa della nonna Madieri poco dopo la mia nascita e vi erano rimasti due anni. Il primo spazio avventuroso della mia vita" (Verde acqua, 3). Sono nitidi pure i ricordi d'infanzia trascorsi in via Angheben in 
cui la scrittrice visse fino all'età di undici anni, quella che cambiò in seguito nome in Zagrebačka ulica e fu il luogo "di giochi sfrenati, di felicità, di libertà" (Verde acqua, 12).

Nascere a Fiume ha avuto e continuerà ad avere per l'autrice un significato particolare e profondo, in quanto il senso di appartenenza a questa città è potenziato da un concetto di cittadinanza che è ed è sempre stato sinonimo di apertura culturale e linguistica, di tolleranza e rispetto reciproco. La lunga tradizione di convivenza armoniosa tra gruppi appartenenti a diverse comunità nazionali ha creato a Fiume un ricco panorama culturale multietnico di cui la tradizione e cultura italiana costituiscono una parte importante. Come sottolinea Claudio Magris,

Fiume è stata un luogo molto più concreto di incontro di culture, incontro che significa anche scontro, conflitto [...] Il senso della nostra esistenza consiste anche nella nostra capacità, quando facciamo i conti con i momenti difficili e duri di un passato senza rimuoverlo, di trasformarlo in qualcosa di vivo e di aperto. (Magris, Fiume, 33-34)

\section{E ancora:}

Quando si parla di una città e una città particolare come Fiume-Rijeka, si parla di qualcosa che non appartiene soltanto alle memorie, al passato, alla nostalgia, bensì di un presente. Noi non siamo qui per nessuna - sia pure legittima, comprensibile - operazione patetica, per guardare indietro, ma per parlare del presente [...] Nel suo libro Verde acqua [...] Marisa Madieri scrive: "Io sono quel vento e quelle rive". Sono non "ero", anche se quando lo scrive non abitava più a Fiume e si era costruita una vita altrove. Noi siamo, ed è questo che ci permette di affrontare il mondo a viso aperto, volto al futuro e non al passato. (Magris, Fiume, 33)

La guerra a Fiume, in quella Fiume multietnica in cui si parlavano quattro lingue - il tedesco, l'ungherese, l'italiano e il croato - fu vissuta agli occhi della bambina Madieri quasi come un'avventura. Persino i continui bombardamenti alla città, le corse nei rifugi, la paura, il dramma della guerra "mi apparivano indecifrabili episodi che non minacciavano ma solo movimentavano la mia vita" (Verde acqua, 12-13).

Il dopoguerra cambiò, di fatto, il volto e l'anima alla sua città natale. Furono anni duri, contraddistinti dalle tragiche vicende degli esuli e dei rimasti, dalle lunghe fila di profughi e di coloro che "partono restando", per parafrasare una frase di Nelida Milani (Bora, 12-13), dell'accorpamento delle classi italiane e croate nelle scuole, dell'estinzione delle sezioni italiane e di conseguenza della chiusura di numerosi istituti scolastici. Marisa 
Madieri ricorda come "La fine della guerra e l'occupazione jugoslava rappresentarono per la mia famiglia un primo periodo di paure, diffidenze, perquisizioni" (Verde acqua, 37). Tra gli italiani dell'istro-quarnerino si diffondeva l'eco della fuga; sembrava che la maggioranza avesse accolto la parola d'ordine "scappare", per cui si verificò il massiccio fenomeno dell'esodo.

È indubbio che le turbolente vicende storiche di questi territori siano un dato di fatto e non possano essere ignorate o storpiate e soprattutto non debbano venir manipolate; e che questa parte del confine orientale sia stata oggetto di scontri e conflitti provocati da diverse ideologie che si sono manifestate nel corso della storia e non solo in quella recente. Franco Fornasaro osserva che

Definire "ciondolo" l'Istria ha un preciso significato: vuol dire essenzialmente tener conto di tutte le oscillazioni storiche, politiche ed etniche che l'hanno interessata, legate ad una geografia che si rivolge in parte verso Venezia, in parte verso il mondo balcanico, o meglio verso il vecchio mondo definibile "illirico" e che si àncora saldamente e nuovamente nel tessuto plurietnico danubiano e mitteleuropeo. Come si vede un quadro d'insieme alquanto complicato in cui i colori sono già stati troppo spesso dati a tinte molto forti e violente. (Fornasaro, 23-24)

Marisa Madieri descrive così la sua partenza da Fiume: "Tra il 1947 e il 1948 a tutti gli italiani rimasti ancora a Fiume fu richiesta l'opzione: bisognava decidere se assumere la cittadinanza jugoslava o abbandonare il paese. La mia famiglia optò per l'Italia e conobbe un anno di emarginazione e persecuzioni” (Verde acqua, 42-43). Nell'estate del 1949 la famiglia Madieri partì da Fiume, mentre il padre, arrestato alcuni mesi prima, fu costretto a rimanere in carcere. Da quel momento inizia per l'autrice il tormentato percorso dell'esule. L'io narrante di Marisa Madieri ne parla il linguaggio, ripercorre la vita misera nei campi profughi come il Silos triestino, descrive il senso di smarrimento e di isolamento a cui gli esuli andarono incontro perché guardati con sospetto dagli abitanti delle città in cui cercarono di rifarsi una vita: "Feci così la mia prima conoscenza del Silos, dove vivevano accampati migliaia di profughi istriani, dalmati o fiumani come noi" (Verde acqua, 67-78). L'autrice ricorda ancora il fetore del Silos, quell'odore indescrivibile che sapeva di dolciastro e stantio e i box in cui erano stipate le famiglie, che non riservavano alcuna intimità. Di quel luogo, vagamente dantesco, l'autrice ricorda anche la violenza subita da una donna istriana, che veniva picchiata dal marito con regolarità una volta al mese. 
L'adattamento alla nuova vita fu lento e non privo di momenti cupi. Le cose cambiarono lievemente con la nuova sistemazione in un modesto alloggio in via Piccardi: il padre, che nel frattempo aveva raggiunto la famiglia a Trieste, aprì un'officina e la madre ritrovò la serenità in una casa tutta sua. Riaffiora alla memoria dell'autrice quel completino color verde acqua, il primo che sua madre era riuscita a comprarle per partecipare a una festa di liceali. Quel cardigan e quella maglia giro collo color verde acqua e quella gonna a campana assumono un valore simbolico, segnano una pagina nuova della sua esistenza e indicano l'inserimento nella nuova struttura sociale:

La mamma mi lesse nel cuore. Portò al Monte di Pietà, come aveva fatto altre volte, il suo braccialetto di metallo bianco e oro, dopo averlo ben strofinato con un panno per renderlo lucido, e la sua pelliccia, probabilmente di coniglio, tutta consunta. Ciò le permise di comperarmi una gonna a campana e un completo formato da un cardigan e una maglia giro collo, in orlon color verde Nilo. Conservai quel completino per anni, con gelosia [...] Anche verde acqua si chiamava quel colore, che per me è ancor oggi il colore dell'amore. (Verde acqua, 122)

Non mancano nel romanzo continui riferimenti alla morte, anche se prevale nel testo l'amore per la vita, vissuta nella quotidianità e nella serenità degli affetti domestici, in cui vengono assaporate la gioie quotidiane, prime tra tutte l'amore del marito e dei figli. Nella sua scrittura caratterizzata dal dire in economia, emerge il suo autentico e sincero amore per la vita, considerato un dono che va conquistato giorno per giorno, in cui la storia va avanti, i fili si riannodano, il tempo rimane e ritorna.

Ernestina Pellegrini definisce Verde acqua "una straordinaria storia di matriarcato [...] di un pietoso ma a volte feroce matriarcato, come è comprensibile per ogni racconto autobiografico che è anche una discesa in fondo al pozzo mitico delle madri" (Pellegrini, 133); in esso emergono donne forti e decise che non si sono piegate alle intemperie, personalità che hanno saputo destreggiarsi abilmente nella vita e che hanno soprattutto saputo inculcare nelle generazioni future un concetto di identità inteso come pluralità culturale e linguistica.

I personaggi femminili che compaiono nel romanzo sono la mamma e le nonne, ben tre: la nonna paterna Madieri, la nonna materna Quarantotto e nonna Anka, l'ultima compagna del padre Luigi Madieri. E in questa storia di matriarcato, in questo intrecciarsi di passato e presente, in cui "sembrava che le nonne Madieri e Quarantotto dessero una mano a nonna Anka, e questa a suor Giovanna che la dava a Nonna Pia Magris, e questa alla zia Nerina e alla zia Nina, e così via fino a Jole, la madre di Marisa" (Pellegrini, 133), Ernestina Pellegrini intravvede l'esigenza dell'autrice di valorizzare la 
saggezza e la pazienza femminili, il criterio nel trasmettere gesti, parole e pensieri che si sedimentano diventando patrimonio culturale e di tradizione. La figura centrale dell'opera è la mamma Jole, alla quale l'autrice si sente profondamente legata e dalla quale ha ereditato i tratti più nascosti della sua personalità: "Le radici della mia forza e della mia capacità di non arrendermi di fronte alle difficoltà affondano nel suo amore" (Verde acqua, 11). Una donna dedita al sacrificio, che ha lottato per il futuro delle proprie figlie, le ha incitate a studiare perché convinta che solo attraverso lo studio sarebbero state in grado di conquistare un futuro migliore e diverso dal suo; un personaggio sottomesso per natura alla famiglia e anche alla madre Maria Quarantotto, della quale si occuperà fino alla morte.

La nonna Madieri era invece una donna forte, saggia, decisa e anticonformista per l'epoca in cui visse. Era nata a Varaždin nel 1868, si chiamava Filippina Mileti e aveva sposato Giorgio Madjari, il nonno di Marisa. Il cognome subì nel tempo delle variazioni: dapprima, Madierich e poi Madieri. Dal matrimonio con questo facoltoso commerciante erano nati parecchi figli, tredici per l'esattezza, ma molti morirono in tenera età. Nel 1904 un evento sconvolse la sua vita: "La leggenda vuole che un giorno nonno Giorgio, avendo perso al Casinò casa, carrozza e cavalli, perdesse anche la moglie la quale, esasperata e incinta dell'ultimo figlio, Luigi, mio padre, abbandonò la famiglia e si recò a Fiume" (Verde acqua, 5). La nonna non si perse d'animo e dapprima fu assunta come donna di pulizie al Casinò, ma grazie alla conoscenza di quattro lingue, ben presto fu promossa a guardarobiera dello stesso Casinò, quel luogo che aveva odiato dal profondo del suo animo. Di questa nonna Marisa Madieri ammira la forza d'animo, il coraggio e anche l'anticonformismo.

La nonna materna Quarantotto, che Ernestina Pellegrini illustra magistralmente paragonandola all'ortica - "noi donne, margherite si nasce e ortiche, magari si diventa, come il personaggio indimenticabile di nonna Quarantotto" (Pellegrini, 133) — aveva una capacità unica di dominare il creato, una vera e propria "libidine di dominio" (Verde acqua, 36). È un personaggio carismatico, dotato di una curiosa intelligenza e vivacità, ma anche di un fascino lievemente malvagio. Nonostante fosse semianalfabeta, fatto che le procurava disagi, questa donna riuscì a ottenere il successo nel suo piccolo mondo, si ritagliò persino un ruolo in prima fila all'interno del Silos. La scrittrice descrive le sue ambizioni unicamente legate a "farsi riverire, temere, servire e, da vecchia, anche venerare come una benefattrice dei profughi e quasi come una santa" (Verde acqua, 29). Della nonna Maria Quarantotto l'autrice non apprezza il suo smisurato desiderio di dominio e la condanna soprattutto per la tirannide esercitata nei confronti della madre Jole. 
Nonna Anka è invece una donna che l'autrice, insieme al marito Claudio e al resto della famiglia, apprezza molto. Anka Grkovi fu vedova Puhalj, vedova Beli, vedova Gregorutti e di fatto anche vedova Madieri in quanto solo a settant'anni era diventata l'ultima compagna di Luigi Madieri, che accanto a lei aveva ritrovato la serenità. Marisa Madieri racconta che Nonna Anka era nata nelle vicinanze di Belgrado, precisamente a Bela Crkva, località che si trova sul confine con la Romania, da madre rumena e padre serbo, e aveva alle spalle tre matrimoni. La descrive come una donna pragmatica: "Si è sempre sposata perseguendo solidi destini patrimoniali e onorevoli sistemazioni sociali e ha servito fedelmente e con scrupolo ogni marito fino alla morte" (Verde acqua, 20). ${ }^{3}$ Marisa Madieri ammira questa donna dal carattere forte e deciso ed è attratta soprattutto dalla visione del mondo di nonna Anka in cui prevale la consapevolezza del fluire della vita.

Le figure femminili descritte in Verde acqua hanno contribuito in maniera determinante alla ricerca e alla costruzione dell'identità dell'autrice e come tali sono fondamentali per comprendere la visione che essa ha della vita e del mondo, sono dei pilastri emozionali sui quali elabora il suo personale approccio agli eventi dell'esistenza: ferma, decisa, stabile, ma anche profondamente emotiva e sensibile.

Verde acqua ha di fatto inaugurato - come abbiamo visto - il filone della letteratura dell'esodo al femminile, cui sono seguite opere significative di altre autrici, incentrate sullo stesso tema. Bora, il libro scritto da Anna Maria Mori e Nelida Milani, la cui pubblicazione risale al 1998, è una di queste. Il libro-epistolario, un'esperienza di vita tra due sponde, è diventato subito un vero e proprio "caso letterario" d'oltreconfine. Compaiono in questa narrazione tutti gli elementi di cui si è trattato finora: la letteratura dell'esodo, le vicende storiche che hanno coinvolto l'Istria, Fiume e la Dalmazia durante e dopo il secondo conflitto mondiale, lo sradicamento dalle proprie origini, la separazione, il trauma della perdita dell'identità come conseguenza dell'esodo e la necessità di un impegno faticoso per affrontare a testa alta il passato, in modo da fronteggiare un futuro sgombro da pesi ideologici, senza però dimenticare mai le proprie origini, la propria lingua e la propria cultura. Sia in Verde acqua che in Bora il tema dell'esodo rappresenta il fulcro della narrazione, ma il modo di raccontarlo è fondamentalmente diverso. In Marisa Madieri il racconto dell'esilio si traduce in pacata consapevolezza nell'intendere la storia della gente di confine, in un'identità di frontiera ormai conciliata, che significa allo stesso tempo fragilità estrema, compenetrazione culturale e linguistica, e imman-

3 Claudio Magris dedica a nonna Anka un capitolo nel suo romanzo Danubio. Milano: Garzanti, 1986, Pp. 309-357. 
cabilmente scontro e conflitto. In Bora non si ritrova questa sensazione di conciliazione, bensì prevalgono rabbia e dolore che puntualmente emergono provocando ulteriori sofferenze.

In questo libro-diario le due autrici ripercorrono il loro vissuto sul filo della memoria tra cambiamenti esterni e soprattutto interiori. Anna Maria Mori lascia la città natale di Pola nel 1945, subito dopo l'arrivo degli Alleati, per addentrarsi nel tormentato cammino dei profughi, portando con sé i ricordi della sua Istria. Nelida Milani, sua coetanea e concittadina, non ha abbandonato la sua città, ma è rimasta — vivendo però lo stesso sradicamento della Mori - nella sua Pola ormai cambiata, in cui parla con timore la sua lingua, si interroga sui tanti perché dell'esistenza e cerca di ricomporre con determinazione e tenacia la sua identità di italiana d'Istria. Il dramma dell'esodo viene narrato di getto dalle due autrici, che dialogando tra di loro riportano in superficie conflitti etnici e politici, memorie condivise, violenze, morti, sofferenze patite, come pure ricordi dell'infanzia, sapori e odori dell'Istria, affetti, amori, insoddisfazioni e successi, espressioni dialettali, cancellazioni della memoria, nomi slavi italianizzati e viceversa e quel silenzioso e cupo fiume di espatriati e di rimasti, entrambi alla ricerca di un posto al sole. Come per Marisa Madieri, l'io narrante della Mori si fa testimone di tutto l'arco della sua esistenza, dalla fanciullezza alla maturità. L'interrogativo che la scrittrice si pone è se sia possibile vivere senza la bambina e il bambino che si è stati, senza il ricordo dell'infanzia e della giovinezza legato a luoghi, fatti, parole e persino "senza la guerra, le bombe, le macerie e il fumo che hanno fatto da cornice a quei tempi lontani, impossibili da dimenticare, pena la cancellazione non dirò di Proust, ma sicuramente di Freud e di tutta la scienza?" (Bora, 5). Questo immenso dolore provocato dell'esodo, questo senso di smarrimento su cui Anna Maria Mori pone l'accento in tutte le pagine del libro, sono equiparati alla fuga, in quanto l'autrice sostiene che da quel momento in poi la sua vita è stata caratterizzata da una fuga continua, uno scappare da sé stessa per non ricordare il dolore provocato dall'esilio, uno stratagemma per evitare il trauma della perdita delle radici e del relativo senso di sicurezza. Questo dolore si è manifestato in tanti modi: uno di questi è stato per esempio la necessità di mentire anche sul suo luogo di nascita, per non riaprire le dolorose ferite sull'esodo.

Fu profuga anche Nelida Milani, che non partì mai, ma rimase nella sua Pola. Le sue parole testimoniano l'altra faccia dell'esodo, l'esilio interno di chi è rimasto; un'altra verità questa che mette a nudo pagine di storia a lungo taciute, ferite che non si sono mai rimarginate, anzi si sono riaperte con l'ultima guerra in questi luoghi, quella che ha portato alla dissoluzione della federazione jugoslava. Bora può essere considerato un esem- 
pio intelligente di scrittura di tipo memorialistico, in cui le due scrittrici rivedono il loro passato, diverso e simile, dove le riflessioni s'intrecciano ai ricordi, gli aneddoti si sovrappongono ai fatti storici e dove il discorso apre molteplici vie alla riflessione.

In questo filone s'inserisce pure il romanzo di Elsa Fonda (la stessa Elsa Fonda che ha interpretato gli adattamenti radiofonici e teatrali de La radura di Marisa Madieri) La cresta sulla zampa di recentissima pubblicazione (Ibiskos, 2010), in cui la memoria individuale dell'autrice si fonde a quella collettiva dell'esodo degli abitanti di Pirano. Un libro impreziosito dalla matrice dialettale, che si avvicina al "raccontarsi" della Madieri in cui si ritrova il tratto nitido della narrazione e del discorso introspettivo. Come osserva Fulvio Senardi, "La cresta sulla zampa di Elsa Fonda va letto come un gesto di riesumazione pietosa a beneficio del presente; nonostante il filo malinconico che si intreccia alla trama, un gesto combattivo di affettuosa fiducia nell'uomo" (Senardi, 5).

Marisa Madieri può considerarsi a pieno titolo la capostipite di questa letteratura, colei che ha ispirato e aperto la strada a un filone che sino a Verde acqua era rimasto sotterraneo e largamente inespresso. I temi che Marisa Madieri elabora nei suoi romanzi e racconti sono variegati e se di primo acchito sembrano essere distinti gli uni dagli altri, in effetti vi è un filo conduttore che li unisce e si tratta proprio del tema dell'esilio. Verde acqua è incentrato sulla questione dell'esodo, La radura ha come protagonista la margherita Dafne, metafora della fragilità della vita, alla ricerca dell'Armonia che non trova. Anche in questo caso parliamo di esodo, ma di fatto si tratta di un esodo interiore "quello della metamorfosi interna che ascolta soltanto la voce dell'anima" (Bianchi, 85). Come tale, La radura della Madieri è un intenso richiamo alla Lichtung, la parola chiave del pensiero di Martin Heidegger. Per il filosofo tedesco la Lichtung, che significa "slargo", indica un modo di essere della verità, ma è anche un termine che richiama la luce. La "radura" con i suoi giochi di chiaro/scuro ci conduce anche nell'universo della filosofa spagnola Maria Zambrano e precisamente nel mondo del suo Chiari del bosco, in cui si traccia il risveglio dell'anima che si incammina per boschi oscuri ${ }^{4}$. In Marisa Madieri è infatti evi-

4 Maria Zambrano (Vélez-Malaga, 1904 - Madrid 1991), filosofa spagnola. Fu allieva di Ortega y Gasset e di Xavier Zubiri. Visse in esilio per circa quarant'anni per sfuggire al regime franchista. Tra le sue numerose opere ricordiamo Chiari del bosco. Milano: Mondadori, 2004; Luoghi della poesia. Milano: Mondadori, 2011; Verso un sapere dell'anima. Milano: Raffaello Cortina Editore, 1996; La confessione come genere letterario. Milano: Mondadori, 1997; Unamuno. Milano: Mondadori, 2006. 
dente questo alternarsi tra chiaro e scuro di cui si serve per rappresentare "il duplice, parallelo scorrere dei punti di vista fondamentali [...] nei confronti della caducità del destino umano" (Pellegrini, 141), una radura brulla in cui coabitano vita e morte. L'autrice non sceglie a caso il titolo di questo breve poema in prosa; in questa "landa stepposa dove l'ambiente non ha nulla di speciale e di attraente" (Bianchi, 54), nasce in un mattino di maggio la margherita Dafne con i suoi "petali spesso arruffati, ma levigati e lucenti, e il bottone, ancora immaturo, turgido e ancora un po' verdino" (La radura, 154). Dafne è una margherita che si affaccia con curiosità alla vita e ne scopre ben presto il suo lato oscuro.

In La conchiglia e altri racconti, ritroviamo ancora il tema dell'esilio nel racconto dell'indigeno che ha perso la donna amata, Naipuni, e nella conchiglia che le aveva donato rivive la sua immagine. Sono attimi singolari per l'indigeno della baia di Tongariki. L'isola è abitata da una popolazione che è stata soggetta a continue migrazioni, costretta a cercare nuovi spazi per sopravvivere. Nel personaggio della vecchia Mauroa, che vive nella grotta di Kalenga dopo aver perso il marito e i figli durante una tempesta, Graziano Bianchi avverte un'ipotetica somiglianza con la figura della nonna Quarantotto per il senso di smarrimento di Mauroa e il suo esilio volontario, eco e metafora dell' esilio narrato dall'autrice in Verde acqua (Bianchi 97).

Marisa Madieri fa rivivere eventi gioiosi della fanciullezza e della giovinezza nella memoria dell'indigeno. Spicca in questo quadro il ricordo della conchiglia donata alla donna amata che è colmo di liricità: il passaggio in cui l'indigeno raccoglie la conchiglia, la porge a Naipuni dai lunghi capelli neri e dalla costituzione esile che lo guarda con occhi lucenti e la avvicina all'orecchio per ascoltarne la musica misteriosa, indicano momenti in cui il registro espressivo della Madieri raggiunge il massimo dell'intensità.

Nel romanzo incompiuto Maria l'autrice affronta invece il tema dell'aborto. Maria Carminati osserva come il romanzo, scritto "per raccontare il dolore delle donne di fronte al dramma della maternità negata, [questa] è forse l'opera più amara di Madieri, quella in cui l'amore per la vita è portato al paradosso di annullarsi per l'incapacità di accoglierla" (Carminati, "Postfazione", 91). In esso l'autrice esprime a chiare lettere la sua posizione sull'aborto; l' "incompiuto catalogo narrativo" (Carminati, "Postfazione", 71) è il prodotto di alcuni interventi critici di Marisa Madieri su questo tema, dei quali il più noto è quello della lettera aperta a Franca Ongaro Basaglia. La protagonista del romanzo proviene da una famiglia pugliese di umili origini completamente sottomessa alla figura autoritaria del padre. Maria fugge da quell'ambiente che le sta stretto e si reca al Nord, precisamente a Padova, dove lavora come bambinaia presso 
una famiglia locale. Da un incontro, di cui si parla poco, ha inizio il dramma della protagonista: "una maternità impossibile che Maria trova la forza - o la dannazione - di negare, di allontanare da sé" (Carminati, "Postfazione", 79). Si conosce ben poco di quanto sia successo effettivamente a Padova e delle modalità dell'interruzione della gravidanza di Maria; quello che si percepisce con precisione sono gli strascichi che questo evento drammatico ha prodotto nell'animo della protagonista. Spiccano nel romanzo le intense pagine rivolte al rapporto con la sorella Sara e al delicato momento in cui questa annuncia la sua prossima maternità. A partire da questo momento prende corpo la narrazione vera e propria, "da qui si svela retrospettivamente il dramma della maternità negata di Maria, da qui riprende senso il fluire della vita [...] la maternità diviene il paradigma stesso dell'accoglienza" (Carminati, "Postfazione”, 84).

Nell'esperienza letteraria dell'autrice, in quei "dolci onesti candidi libri” (Pellegrini, 139) nei quali si spazia tra il passato e il presente, tra il reale e l'immaginario, prevale sempre e comunque l'amore per la vita. La narrativa di Marisa Madieri si può pertanto raffigurare nel

rapporto fra ciò che contiene e ciò che è contenuto, sia questo l'esperienza passata che si è depositata e organizzata a frammenti nella memoria e poi nell'opera letteraria, sia questo lo sguardo da bambino, puro e selvaggio (della margherita della Radura e poi de Il bambino con le ali, nel racconto omonimo) - con i suoi sogni, le sue paure, le sue speranze e illusioni - e lo sguardo eminentemente pratico e addomesticato delle matrone di Verde acqua. (Pellegrini, 142)

Il 7 aprile 2010, Marisa Madieri ha compiuto simbolicamente il suo viaggio di ritorno a Itaca. La città di Fiume le ha reso omaggio ponendo una lapide sulla sua casa natale. Alla cerimonia erano presenti familiari e autorità. Dopo la cerimonia ha avuto luogo, presso la Comunità degli Italiani di Fiume, una serata dedicata all'autrice cui hanno partecipato Claudio Magris e Giacomo Scotti. Claudio Magris, in un'intervista rilasciata per l'occasione alla rivista culturale fiumana "La Battana", rileva la semplicità della scrittura madieriana, sottolineata molto spesso dalla critica:

Anzitutto l'essenzialità faceva parte dell'intero suo stile di vita, di espressione, anche nel parlare quotidiano, nell'affrontare e risolvere i problemi e così via. Era maestra nell'arte di 'levare', in quella capacità di affidare al non-detto la forza evocativa che fa sorgere tutto un mondo, molto più ricco e vivo e vario che non una descrizione dettagliata e completa, che rischia di distruggere la fantasia. (Magris, "Omaggio a Marisa Madieri", 
Lautrice amava scherzosamente ribadire come questa sua concisione nell'espressione fosse una sorta d'unione che le derivava dalla sintassi latina e dalle lettere d'ufficio che scriveva mentre era impiegata alle Assicurazioni Generali.

L'importanza dell'iniziativa del comune di Fiume è stata confermata dallo scrittore fiumano Giacomo Scotti, il quale ha sottolineato che "con la sua opera, Marisa Madieri è tornata ad essere fiumana ed ha acquisito anche la cittadinanza letteraria croata" (Scotti, 3); il suo simbolico ritorno a Fiume è un'ulteriore conferma di quell'aspetto conciliante e composito della frontiera troppo spesso sottotaciuto. La letteratura dell'esodo ci ha lasciato validissimi esempi di come un confine serva anche a non dividere: Marisa Madieri, Fulvio Tomizza, Osvaldo Ramous, Diego Zandel, Graziella Fiorentin, Anna Maria Mori, Elsa Fonda, Ester Barlessi, Nelida Milani, indicano un percorso che avvia alla comprensione della multiculturalità, in cui il confine orientale viene privato della sua componente esclusivamente divisoria.

Marisa Madieri si è impegnata ad affrontare a testa alta e a viso aperto il passato per fronteggiare un futuro sgombro da pesi ideologici, senza dimenticare mai le proprie origini, la propria lingua, la propria cultura e soprattutto l'aspetto plurimo dell'identità di frontiera. Il suo impegno letterario di alto valore artistico rappresenta pertanto anche una indiscutibile e difficilmente superabile ricostruzione di un capitolo di storia a lungo sottaciuto.

\section{UniVERSiTÀ DEGLI STUdI Di FIUME}

\section{OPERE CITATE}

AA.VV. La forza della fragilità. A cura di E. Deghenghi Olujić. Fiume: Edit, 2004. Benelli, Graziano. "La prima fortuna di 'Verde acqua'." Il lettore di provincia 72 (settembre 1988): 36-48.

Benussi, Cristina. "Storie e memorie letterarie." La Battana 160 (aprile-giugno 2006): 31-65.

Bianchi, Graziano. La narrativa di Marisa Madieri. Firenze: Le lettere, 2003.

Carminati, Maria. "Postfazione" a Madieri, Marisa. Maria. Milano: Archinto, 2007. 71-91.

. "Marisa Madieri e la letteratura femminile dell'esodo." La Battana 160 (aprile-giugno 2006): 175-191.

Fonda, Elsa. La cresta sulla zampa. Empoli: Ibiskos Editrice Risolo, 2010.

Fornasaro, Franco. "Il ciondolo etnico istriano: cent'anni di cambiamenti." Etnie a confronto. Il mosaico Europa. Mestre-Venezia: Alcione Editore, 1997. 23-31. Madieri, Marisa. "Due voci sulla liberazione della donna. Aborto sì, aborto no. 
Discussione con Franca Ongaro Basaglia.” Nuova Società X.221 (1982): 5963.

Acqua è poesia - Water is poetry. Ciga Hotels Magazine XVII.81 (1989): 6471.

"Emarginazione a Trieste: famiglia." Giornate del Volontariato 2-5 dicembre 1989 sul tema 'Comunicare la solidarietà.' A cura della Consulta del Volontariato di Trieste e del Movimento di Volontariato Italiano MO. V. I. 1989: 14-19.

"Introduzione" a 'Ambito A - Gruppo di studio 4 - Accoglienza della vita nascente e iniziative per una efficace cultura della vita: associazionismo, volontariato e chiesa locale." A servizio della vita umana. A cura della CEI. Roma: A.V.E., 1990.

- Verde acqua, La radura e altri racconti. Torino: Einaudi, 2006. Maria. A cura di Maria Carminati. Milano: Archinto, 2007.

Magris, Claudio. Danubio. Milano: Garzanti, 1986. . "Introduzione" a AA.VV. Fiume crocevia di popoli e culture. Atti del Convegno internazionale. Roma, 27 ottobre 2005. A cura di Giovanni Stelli. Roma: Collana di studi storici fiumani, 2006. 33-34.

"Omaggio a Marisa Madieri. Intervista a Claudio Magris.” A cura di Patrizia Venucci Merdžo. La Battana 176 (marzo-giugno 2010): 75-79.

Maier, Bruno. La letteratura italiana dell'Istria dalle origini al Novecento. Trieste: Edizioni Italo Svevo, 1996.

Mori, Anna Maria e Milani, Nelida. Bora. Piacenza: Frassinelli, 1998.

Moscarda Mitrović, Eliana. "Marisa Madieri: Verde acqua." La forza della fragilità. A cura di E. Deghenghi Olujić.Vol. 1. Fiume: Edit, 2004. 191-210.

Paccagnini, Ermanno. "Introduzione" a Madieri, Marisa. Verde acqua. La radura e altri racconti. Torino: Einaudi, 2006. v-xxi.

Pellegrini, Ernestina. “'La radura' di Marisa Madieri. Le radici della nostra debolezza.” Le città interiori. Bergamo: Moretti \& Vitali, 1995. 131-155.

Senardi, Fulvio. "Elsa Fonda: sul crinale più alto della letteratura dell'esodo. La cresta sulla zampa." Trieste Arte\&Cultura 5 151/154 (settembre/dicembre 2010): 5.

Scotti, Giacomo. "Un profilo umano e letterario. Marisa Madieri". Trieste ArtedCultura 5 151/154 (settembre/dicembre 2010): 2-3.

Zambrano, Maria. Chiari del bosco. Milano: Mondadori, 2004. 
